\title{
Anti-BRST in the Causal Approach
}

\author{
Dan-Radu Grigore
}

check for updates

Citation: Grigore, D.-R. Anti-BRST in the Causal Approach. Universe 2021, 7, 26. https://doi.org/10.3390/ universe7020026

Academic Editors: Sayantan Choudhury, Sudhakar Panda and Subhashish Banerjee Received: 25 December 2020 Accepted: 20 January 2021 Published: 26 January 2021

Publisher's Note: MDPI stays neutral with regard to jurisdictional clai$\mathrm{ms}$ in published maps and institutional affiliations.

Copyright: (C) 2021 by the author. Licensee MDPI, Basel, Switzerland. This article is an open access article distributed under the terms and conditions of the Creative Commons Attribution (CC BY) license (https:// creativecommons.org/licenses/by/ $4.0 /)$.
Department of Theoretical Physics, Institute for Physics and Nuclear Engineering "Horia Hulubei", P.O. Box MG 6, 077125 Bucharest-Măgurele, Romania; grigore@theory.nipne.ro

\begin{abstract}
It is known that the elimination of anomalies in all orders of perturbation theory is an open problem. The constraints given by usual invariance properties and the Wess-Zumino identities are not enough to eliminate the anomalies in the general case of a Yang-Mills theory. So, any new symmetry of the model could restrict further the anomalies and be a solution of the problem. We consider the anti-BRST transform of Ojima in the causal approach and investigate if such new restrictions are obtained. Unfortunately, the result is negative: if we have BRST invariance up to the second order of perturbation theory, we also have anti-BRST invariance up to the same order. Probably, this result is true in all orders of perturbation theory. So, anti-BRST transform gives nothing new, and we have to find other ideas to restrict and eventually eliminate the anomalies for a general Yang-Mills theory.
\end{abstract}

Keywords: perturbative quantum field theory; causal approach

\section{Introduction}

The general framework of perturbation theory consists of the construction of some distribution-valued operators called chronological products [1]. We prefer the framework from [2]: for every set of Wick monomials $W_{1}\left(x_{1}\right), \ldots, W_{n}\left(x_{n}\right)$ acting in some Fock space $\mathcal{H}$ one associates the distribution-valued operator $T\left(W_{1}\left(x_{1}\right), \ldots, W_{n}\left(x_{n}\right)\right) \equiv$ $T^{W_{1}, \ldots, W_{n}}\left(x_{1}, \ldots, x_{n}\right)$ such that a set of axioms, essentially proposed by Bogoliubov, are verified. The modern construction of the chronological products can be done recursively according to the Epstein-Glaser prescription [3-7] (which reduces the induction procedure to a distribution splitting of some distributions with causal support) or according to the Stora prescription [8] (which reduces the renormalization procedure to the process of extension of distributions). These products are not uniquely defined but there are some natural limitations on the arbitrariness. If the arbitrariness does not grow with $n$ (the order of perturbation theory) we have a renormalizable theory. An equivalent point of view uses retarded products [9].

In this paper we will use the causal approach to perturbative quantum field theory: this in fact the Epstein-Glaser prescription adapted to gauge models by G. Scharf and collaborators. The literature is quite extensive: a number of books had appeared $[5,6,10,11]$ and also some review papers [12-15].

The description of higher spins in perturbation theory can be problematic. If we describe them by fields carrying only physical degrees of freedom, then the theories are usually not renormalizable. However, one can save renormalizability using ghost fields. Such theories are defined in a Fock space $\mathcal{H}$ with indefinite metric, generated by physical and un-physical fields (called ghost fields). One selects the physical states assuming the existence of an operator $Q$ called gauge charge which verifies $Q^{2}=0$ and such that the physical Hilbert space is by definition $\mathcal{H}_{\text {phys }} \equiv \operatorname{Ker}(Q) / \operatorname{Im}(Q)$. The fact that two distinct mathematical states from $\mathcal{H}$ can be associated to the same physical context is called gauge freedom and the corresponding theories are called gauge theories. The graded commutator $d_{Q}$ of the gauge charge with any operator $A$ of fixed ghost number

$$
d_{Q} A=[Q, A]
$$


(where $[\cdot, \cdot]$ denotes the graded commutator) verifies

$$
d_{Q}^{2}=0
$$

so $d_{Q}$ is a co-chain operator in the space of Wick polynomials.

A gauge theory assumes also that there exists a Wick polynomial of null ghost number $T(x)$ called the interaction Lagrangian such that

$$
d_{Q} T=i \partial_{\mu} T^{\mu}
$$

for some other Wick polynomials $T^{\mu}$. This relation means that the expression $T$ leaves invariant the physical states, at least in the adiabatic limit. Indeed, we have:

$$
T(f) \mathcal{H}_{\text {phys }} \subset \mathcal{H}_{\text {phys }}
$$

up to terms which can be made as small as desired (making the test function $f$ flatter and flatter, i.e., $f$ is approaching 1 in the distributional sense). In all known models one finds out that there exists a chain of Wick polynomials $T^{\mu}, T^{[\mu v]}, T^{[\mu v \rho]}, \ldots$ such that:

$$
d_{Q} T=i \partial_{\mu} T^{\mu}, \quad d_{Q} T^{\mu}=i \partial_{v} T^{[\mu v]}, \quad d_{Q} T^{[\mu v]}=i \partial_{\rho} T^{[\mu v \rho]}, \ldots
$$

where the brackets express completely antisymmetric in all indexes; it follows that the chain of relations stops after a finite number of steps. We can also use a compact notation $T^{I}$ where $I$ is a collection of indexes $I=\left[v_{1}, \ldots, v_{p}\right](p=0,1, \ldots$,$) and one can write$ compactly the relations (5) as follows:

$$
d_{Q} T^{I}=i \partial_{\mu} T^{I \mu}
$$

All these polynomials have the same canonical dimension

$$
\omega\left(T^{I}\right)=\omega_{0}, \forall I
$$

and the ghost number:

$$
g h\left(T^{I}\right)=|I| .
$$

If the interaction Lagrangian $T$ is Lorentz invariant, then one can prove that the expressions $T^{I},|I|>0$ can be taken Lorentz covariant.

Now we can construct the chronological products

$$
T^{I_{1}, \ldots, I_{n}}\left(x_{1}, \ldots, x_{n}\right) \equiv T\left(T^{I_{1}}\left(x_{1}\right), \ldots, T^{I_{n}}\left(x_{n}\right)\right)
$$

according to the recursive procedure. We say that the theory is gauge invariant in all orders of perturbation theory if the following set of identities generalizing (6):

$$
d_{Q} T^{I_{1}, \ldots, I_{n}}=i \sum_{l=1}^{n}(-1)^{s_{l}} \frac{\partial}{\partial x_{l}^{\mu}} T^{I_{1}, \ldots, I_{l} \mu, \ldots, I_{n}}
$$

are true for all $n \in \mathbb{N}$ and all $I_{1}, \ldots, I_{n}$. Here we have defined

$$
s_{l} \equiv \sum_{j=1}^{l-1}|I|_{j} .
$$

Such identities can be usually broken by anomalies, i.e., expressions of the type $A^{I_{1}, \ldots, I_{n}}$ which are quasi-local and might appear in the right-hand side of the relation (10). These anomalies are constrained by some identities (the Wess-Zumino relations). It is still an unsolved problem to prove that the anomalies can be eliminated by convenient 
redefinitions of the chronological products. This problem is not resolved satisfactorly in other approaches also, at least in our opinion.

One idea to eliminate the anomalies is to try to find new restrictions verified by them beside the Wess-Zumino relations. One possibility could be the anti-BRST transform introduced in [16]. We will prove in this paper a negative result, namely that up to the second order of perturbation theory, the anti-BRST transform does not produce new constraints on the model; that is if we impose BRST symmetry up to the second order, we automatically have anti-BRST up to the second order.

We should mention that the literature is rather extensive on the BRST transform in the functional formalism, based on the master equation. There are some differences between the two approaches and there is no proof that they are equivalent. We only mention [17] where a result of the same nature as ours is proved, namely that BRST and anti-BRST transforms are connected by the Hodge map. For the treatment of anomalies in the functional formalism we cite $[18,19]$.

In the next section, we will briefly present the Yang-Mills model in our preferred compact notations. In Section 3, we prove our main result.

\section{Yang-Mills Models in the Causal Formalism}

We give some results from [20].

\subsection{Massless Particles of Spin 1 (Photons)}

We consider a vector space $\mathcal{H}$ of Fock type generated (in the sense of Borchers theorem) by the vector field $v_{\mu}$ (with Bose statistics) and the scalar fields $u, \tilde{u}$ (with Fermi statistics). The Fermi fields are usually called ghost fields. We suppose that all these (quantum) fields are of null mass. Let $\Omega$ be the vacuum state in $\mathcal{H}$. In this vector space we can define a sesquilinear form $\langle\cdot, \cdot\rangle$ in the following way: the (non-zero) 2-point functions are by definition:

$$
\begin{array}{r}
<\Omega, v_{\mu}\left(x_{1}\right) v_{\mu}\left(x_{2}\right) \Omega>=i \eta_{\mu \nu} D_{0}^{(+)}\left(x_{1}-x_{2}\right), \\
<\Omega, u\left(x_{1}\right) \tilde{u}\left(x_{2}\right) \Omega>=-i D_{0}^{(+)}\left(x_{1}-x_{2}\right) \quad<\Omega, \tilde{u}\left(x_{1}\right) u\left(x_{2}\right) \Omega>=i D_{0}^{(+)}\left(x_{1}-x_{2}\right)
\end{array}
$$

and the $n$-point functions are generated according to Wick theorem. Here $\eta_{\mu v}$ is the Minkowski metrics (with diagonal $1,-1,-1,-1$ ) and $D_{0}^{(+)}$is the positive frequency part of the Pauli-Jordan distribution $D_{0}$ of null mass. To extend the sesquilinear form to $\mathcal{H}$ we define the conjugation by

$$
v_{\mu}^{\dagger}=v_{\mu}, \quad u^{\dagger}=u, \quad \tilde{u}^{\dagger}=-\tilde{u} .
$$

Now we can define in $\mathcal{H}$ the operator $Q$ according to the following formulas:

$$
\begin{aligned}
& {\left[Q, v_{\mu}\right]=i \partial_{\mu} u, \quad[Q, u]=0, \quad[Q, \tilde{u}]=-i \partial_{\mu} v^{\mu}} \\
& Q \Omega=0
\end{aligned}
$$

where by $[\cdot, \cdot]$ we mean the graded commutator. One can prove that $Q$ is well defined: basically it leaves invariant the causal commutation relations. The usefulness of this construction follows from:

Theorem 1. The operator $Q$ verifies $Q^{2}=0$. The factor space $\operatorname{Ker}(Q) / \operatorname{Ran}(Q)$ is isomorphic to the Fock space of particles of zero mass and helicity 1 (photons).

\subsection{Massive Particles of Spin 1 (Heavy Bosons)}

We repeat the whole argument for the case of massive photons, i.e., particles of spin 1 and positive mass. 
We consider a vector space $\mathcal{H}$ of Fock type generated by the vector field $v_{\mu}$, the scalar field $\Phi$ (with Bose statistics) and the scalar fields $u, \tilde{u}$ (with Fermi statistics). We suppose that all these (quantum) fields are of mass $m>0$. In this vector space we can define a sesquilinear form $\langle\cdot, \cdot\rangle$ in the following way: the (non-zero) 2-point functions are by definition:

$$
\begin{array}{rr}
<\Omega, v_{\mu}\left(x_{1}\right) v_{\mu}\left(x_{2}\right) \Omega>=i \eta_{\mu v} D_{m}^{(+)}\left(x_{1}-x_{2}\right), \quad<\Omega, \Phi\left(x_{1}\right) \Phi\left(x_{2}\right) \Omega>=-i D_{m}^{(+)}\left(x_{1}-x_{2}\right) \\
<\Omega, u\left(x_{1}\right) \tilde{u}\left(x_{2}\right) \Omega>=-i D_{m}^{(+)}\left(x_{1}-x_{2}\right), \quad<\Omega, \tilde{u}\left(x_{1}\right) u\left(x_{2}\right) \Omega>=i D_{m}^{(+)}\left(x_{1}-x_{2}\right)
\end{array}
$$

and the $n$-point functions are generated according to Wick theorem. Here $D_{m}^{(+)}$is the positive frequency part of the Pauli-Jordan distribution $D_{m}$ of mass $m$. To extend the sesquilinear form to $\mathcal{H}$ we define the conjugation by

$$
v_{\mu}^{\dagger}=v_{\mu}, \quad u^{\dagger}=u, \quad \tilde{u}^{\dagger}=-\tilde{u}, \quad \Phi^{\dagger}=\Phi .
$$

Now we can define in $\mathcal{H}$ the operator $Q$ according to the following formulas:

$$
\begin{array}{r}
{\left[Q, v_{\mu}\right]=i \partial_{\mu} u, \quad[Q, u]=0, \quad[Q, \tilde{u}]=-i\left(\partial_{\mu} v^{\mu}+m \Phi\right) \quad[Q, \Phi]=i m u,} \\
Q \Omega=0 .
\end{array}
$$

One can prove that $Q$ is well defined. We have a result similar to the first theorem of this section:

Theorem 2. The operator $Q$ verifies $Q^{2}=0$. The factor space $\operatorname{Ker}(Q) / \operatorname{Ran}(Q)$ is isomorphic to the Fock space of particles of mass $m$ and spin 1 (massive photons).

\subsection{The Generic Yang-Mills Case}

The situations described above (of massless and massive photons) are susceptible of the following generalizations. We can consider a system of particles of null mass and helicity 1 using the idea of the first subsection for triplets $\left(v_{a}^{\mu}, u_{a}, \tilde{u}_{a}\right), a \in I_{1}$ of massless fields; here $I_{1}$ is a set of indexes. All the relations have to be modified by appending an index $a$ to all these fields.

In the massive case we have to consider quadruples $\left(v_{a}^{\mu}, u_{a}, \tilde{u}_{a}, \Phi_{a}\right), a \in I_{2}$ of fields of mass $m_{a}$ and use the second subsection; here $I_{2}$ is another set of indexes.

We can consider now the most general case involving fields of spin not greater that 1. We take $I=I_{1} \cup I_{2} \cup I_{3}$ a set of indexes and for any index we take a quadruple $\left(v_{a}^{\mu}, u_{a}, \tilde{u}_{a}, \Phi_{a}\right), a \in I$ of fields with the following conventions: (a) For $a \in I_{1}$ we impose $\Phi_{a}=0$ and we take the masses to be null $m_{a}=0$; (b) For $a \in I_{2}$ we take the all the masses strictly positive: $m_{a}>0$; (c) For $a \in I_{3}$ we take $v_{a}^{\mu}, u_{a}, \tilde{u}_{a}$ to be null and the fields $\Phi_{a} \equiv \phi_{a}^{H}$ of mass $m_{a}^{H} \geq 0$. The fields $\phi_{a}^{H}$ are called Higgs fields.

If we define $m_{a}=0, \forall a \in I_{3}$ then we can define in $\mathcal{H}$ the operator $Q$ according to the following formulas for all indexes $a \in I$ :

$$
\begin{array}{r}
{\left[Q, v_{a}^{\mu}\right]=i \partial^{\mu} u_{a},\left[Q, u_{a}\right]=0,} \\
{\left[Q, \tilde{u}_{a}\right]=-i\left(\partial_{\mu} v_{a}^{\mu}+m_{a} \Phi_{a}\right) \quad\left[Q, \Phi_{a}\right]=i m_{a} u_{a},} \\
Q \Omega=0 .
\end{array}
$$

If we consider matter fields also, i.e., some set of Dirac fields with Fermi statistics: $\psi_{A}, A \in I_{4}$ then we impose

$$
d_{Q} \psi_{A}=0
$$




\subsection{The Yang-Mills Interaction}

In the framework and notations from the end of the preceding subsection we have the following result which describes the most general form of the Yang-Mills interaction [21-23]. Summation over the dummy indexes is used everywhere.

Theorem 3. Let $T$ be a relative cocycle for $d_{Q}$, i.e., verifies (3) and also: (1) is tri-linear in the fields; (2) is of canonical dimension $\omega(T) \leq 4 ; 3$ ) has ghost number $g h(T)=0$. Then:

(i) $T$ is (relatively) cohomologous to a non-trivial co-cycle of the form:

$$
\begin{array}{r}
T=f_{a b c}\left(\frac{1}{2} v_{a \mu} v_{b v} F_{c}^{\nu \mu}+u_{a} v_{b}^{\mu} \partial_{\mu} \tilde{u}_{c}\right) \\
+f_{a b c}^{\prime}\left(\Phi_{a} \partial_{\mu} \Phi_{b} v_{c}^{\mu}-m_{b} \Phi_{a} v_{b}^{\mu} v_{c \mu}+m_{b} \Phi_{a} \tilde{u}_{b} u_{c}\right) \\
+\frac{1}{3 !} f_{a b c}^{\prime \prime} \Phi_{a} \Phi_{b} \Phi_{c}+j_{a}^{\mu} v_{a \mu}+j_{a} \Phi_{a} ;
\end{array}
$$

where we can take the constants $f_{a b c}=0$ if one of the indexes is in $I_{3} ;$ also $f_{a b c}^{\prime}=0$ if $c \in I_{3}$ or one of the indexes $a$ and $b$ are from $I_{1} ;$ and $j_{a}^{\mu}=0$ if $a \in I_{3} ; j_{a}=0$ if $a \in I_{1}$. Moreover we have:

(a) The constants $f_{a b c}$ are completely antisymmetric

$$
f_{a b c}=f_{[a b c]} .
$$

(b) The expressions $f_{a b c}^{\prime}$ are antisymmetric in the indexes $a$ and $b$ :

$$
f_{a b c}^{\prime}=-f_{b a c}^{\prime}
$$

and are connected to $f_{a b c}$ by:

$$
f_{a b c} m_{c}=f_{c a b}^{\prime} m_{a}-f_{c b a}^{\prime} m_{b} .
$$

(c) The (completely symmetric) expressions $f_{a b c}^{\prime \prime}=f_{\{a b c\}}^{\prime \prime}$ verify

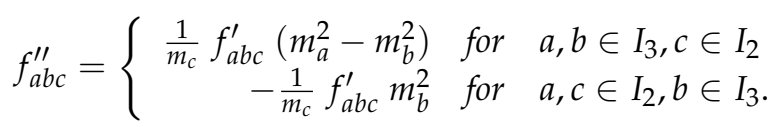

(d) the expressions $j_{a}^{\mu}$ and $j_{a}$ are bilinear in the Fermi matter fields: in tensor notations;

$$
j_{a}^{\mu}=\sum_{\epsilon} \bar{\psi} t_{a}^{\epsilon} \otimes \gamma^{\mu} \gamma_{\epsilon} \psi \quad j_{a}=\sum_{\epsilon} \bar{\psi} s_{a}^{\epsilon} \otimes \gamma_{\epsilon} \psi
$$

where for every $\epsilon= \pm$ we have defined the chiral projectors of the algebra of Dirac matrices $\gamma_{\epsilon} \equiv \frac{1}{2}\left(I+\epsilon \gamma_{5}\right)$ and $t_{a}^{\epsilon}, s_{a}^{\epsilon}$ are $\left|I_{4}\right| \times\left|I_{4}\right|$ matrices. If $M$ is the mass matrix $M_{A B}=\delta_{A B} M_{A}$ then we must have

$$
\partial_{\mu} j_{a}^{\mu}=m_{a} j_{a} \quad \Leftrightarrow \quad m_{a} s_{a}^{\epsilon}=i\left(M t_{a}^{\epsilon}-t_{a}^{-\epsilon} M\right) .
$$

(ii) The relation $d_{Q} T=i \partial_{\mu} T^{\mu}$ is verified by:

$$
T^{\mu}=f_{a b c}\left(u_{a} v_{b v} F_{c}^{v \mu}-\frac{1}{2} u_{a} u_{b} \partial^{\mu} \tilde{u}_{c}\right)+f_{a b c}^{\prime}\left(\Phi_{a} \partial^{\mu} \Phi_{b} u_{c}-m_{b} \Phi_{a} v_{b}^{\mu} u_{c}\right)+j_{a}^{\mu} u_{a}
$$

(iii) The relation $d_{Q} T^{\mu}=i \partial_{v} T^{\mu v}$ is verified by:

$$
T^{\mu v} \equiv \frac{1}{2} f_{a b c} u_{a} u_{b} F_{c}^{\mu \nu}
$$


Now if we impose gauge invariance in the second order of perturbation theory, i.e., (10) for $n=2$ we get new constraints on the constants from the interaction Lagrangian (for an extensive treatment see [20-23]):

$$
\sum_{c}\left(f_{a b c} f_{d e c}+f_{b d c} f_{a e c}+f_{d a c} f_{b e c}\right)=0
$$

(Jacobi identity)

$$
\sum_{c}\left[f_{d c a}^{\prime} f_{c e b}^{\prime}-(a \leftrightarrow b)\right]=-\sum_{c} f_{a b c} f_{d e c}^{\prime}, \quad a, b \in I_{1} \cup I_{2}, d, e \in I_{2} \cup I_{3}
$$

(the representation property for the Bose sector)

$$
\left[t_{a}^{\epsilon}, t_{b}^{\epsilon}\right]=i f_{a b c} t_{c}^{\epsilon}
$$

(the representation property for the Fermi sector)

$$
t_{a}^{-\epsilon} s_{b}^{\epsilon}-s_{b}^{\epsilon} t_{a}^{\epsilon}=i f_{b c a}^{\prime} s_{c}^{\epsilon}
$$

(a tensor representation property for the expressions $s_{a}$ ).

This result was obtained independently in $[22,23]$ and $[24,25]$, respectively. It gives a new perspective on Higgs mechanism; some new references on this subject are [26-28].

\subsection{Causal Perturbation Theory}

We give the idea of the construction of the chronological products only in the second order of perturbation theory, relevant for our result. The basic construction of Epstein and Glaser is the construction of the causal commutator. More details can be found in [20]. We mention the basic procedure used in the causal approach. If we want to construct the chronological product $T\left(A\left(x_{1}\right), B\left(x_{2}\right)\right)$ for arbitrary Wick monomials $A$ and $B$, the idea of Epstein and Glaser is to consider the (graded) commutator

$$
D\left(x_{1}, x_{2}\right) \equiv\left[A\left(x_{1}\right), B\left(x_{2}\right)\right] .
$$

It is easy to see that the tree contribution to this commutator is of the form:

$$
D_{(0)}\left(x_{1}, x_{2}\right)=\sum p_{j}(\partial) D\left(x_{1}-x_{2}\right) W_{j}\left(x_{1}, x_{2}\right)
$$

where $p_{j}$ are polynomials in the partial derivatives and $W_{j}$ are Wick polynomials. Then one can obtain the associated chronological products if one makes in the preceding formula the substitution $D \rightarrow D^{F}$ i.e., if we replace the Pauli-Villars causal distribution with the Feynman propagator:

$$
T_{(0)}\left(x_{1}, x_{2}\right)=\sum p_{j}(\partial) D^{F}\left(x_{1}-x_{2}\right) W_{j}\left(x_{1}, x_{2}\right) .
$$

In this way we fulfill Bogoliubov axioms in the second order for the tree contributions. However, in this way we can produce anomalies in the relation (10). The reason is that for the gauge invariance of the causal commutators-e.g., relation (55) from the next section we need the Klein-Gordon equation for the Pauli-Villars distribution $\left(\square+m^{2}\right) D_{m}=0$ but for the gauge invariance of the chronological products we must use $\left(\square+m^{2}\right) D_{m}^{F}=\delta$. In some cases it is possible to eliminate these anomalies if we use finite renormalizations

$$
T_{(0)}\left(x_{1}, x_{2}\right) \rightarrow T_{(0)}^{\mathrm{ren}}\left(x_{1}, x_{2}\right) \equiv T_{(0)}\left(x_{1}, x_{2}\right)+N\left(x_{1}, x_{2}\right)
$$

where $N$ are finite renormalizations; they must be quasi-local expressions i. e they are of the form $q_{j}(\partial) \delta\left(x_{1}-x_{2}\right) N_{j}\left(x_{1}\right)$ with $N_{j}$ Wick polynomials. This program can be extended, in principle to loop contributions and to higher orders of perturbation theory, but a complete analysis is not available now. 


\section{Anti-BRST Transform}

In Section 2, we have introduced the BRST transform; we prefer to rewrite it using the (graded) commutator $d_{Q}$ :

$$
d_{Q} v_{a}^{\mu}=i \partial^{\mu} u_{a}, \quad d_{Q} u_{a}=0, \quad d_{Q} \tilde{u}_{a}=-i\left(\partial_{\mu} v_{a}^{\mu}+m \Phi_{a}\right), \quad \begin{array}{r}
d_{Q} \Phi_{a}=i m_{a} u_{a} \\
d_{Q} \psi_{A}=0 .
\end{array}
$$

These expressions are the linear part of the non-linear BRST transform from the classical field theory. We proceed in the same spirit with the anti-BRST transform from [16] and obtain the operator $d_{Q}^{\text {anti }}$ (associated to the anti-BRST transform $Q_{\text {anti }}$ ) and given by:

$$
\begin{array}{r}
d_{Q}^{\mathrm{anti}} v_{a}^{\mu}=i \partial^{\mu} \tilde{u}_{a}, \quad d_{\mathrm{Q}}^{\mathrm{anti}} u_{a}=i\left(\partial_{\mu} v_{a}^{\mu}+m \Phi_{a}\right), \quad d_{\mathrm{Q}}^{\mathrm{anti}} \tilde{u}_{a}=0, \quad d_{\mathrm{Q}}^{\mathrm{anti}} \Phi_{a}=i m_{a} \tilde{u}_{a}, \\
d_{Q}^{\text {anti }} \psi_{A}=0,
\end{array}
$$

and

$$
Q_{\text {anti }} \Omega=0 .
$$

As in the case of the BRST transform (37), we immediately check that

$$
\left(Q_{\text {anti }}\right)^{2}=0 \quad \Longleftrightarrow \quad\left(d_{Q}^{\text {anti }}\right)^{2}=0 .
$$

We also have

$$
\left\{Q, Q_{\text {anti }}\right\}=0 \text {. }
$$

Now we want to investigate if a theorem similar to 3 is valid for the anti-BRST transform. Indeed we have:

Theorem 4. Suppose that the conditions from theorem 3 are fulfilled. Then:

(i) The relation $d_{Q}^{\text {anti }} T=i \partial_{\mu} T_{\text {anti }}^{\mu}$ is verified for

$$
\begin{array}{r}
T_{\text {anti }}^{\mu}=f_{a b c}\left(\tilde{u}_{a} v_{b v} F_{c}^{v \mu}+\tilde{u}_{a} \partial_{v} v_{b}^{v} v_{c}^{\mu}-\right. \\
\left.-u_{a} \tilde{u}_{b} \partial^{\mu} \tilde{u}_{c}+\frac{1}{2} \partial^{\mu} u_{a} \tilde{u}_{b} \tilde{u}_{c}-m_{c} \tilde{u}_{a} v_{b}^{\mu} \Phi_{c}\right) \\
+f_{a b c}^{\prime}\left(\Phi_{a} \partial^{\mu} \Phi_{b} \tilde{u}_{c}-m_{b} \Phi_{a} v_{b}^{\mu} \tilde{u}_{c}\right)+j_{a}^{\mu} \tilde{u}_{a}
\end{array}
$$

(ii) The relation $d_{\mathrm{Q}}^{\mathrm{anti}} T_{\mathrm{anti}}^{\mu}=i \partial_{\nu} T_{\mathrm{anti}}^{\mu v}$ is verified by:

$$
T_{\text {anti }}^{\mu \nu} \equiv \frac{1}{2} f_{a b c} \tilde{u}_{a} \tilde{u}_{b} F_{c}^{\mu v} .
$$

Proof. It is elementary, by direct computations. We must cleverly use all the linear relations between the constants of $T$ derived in Theorem 3. mation

We remark that the expression $T_{\text {anti }}^{\mu v}$ can be obtained from $T^{\mu v}$ if we make the transfor-

$$
u_{a} \longleftrightarrow-\tilde{u}_{a}
$$

which also preserves the canonical anti-commutation relations and maps $Q$ in $Q_{\mathrm{anti}}$; however this is not true for $T^{\mu} \longleftrightarrow T_{\text {anti }}^{\mu}$.

The preceding result shows that, as in [16], we get nothing new from anti-BRST in the first order of perturbation theory. We investigate now if the same is true for the second order of perturbation theory. We are interested to impose gauge invariance for the anti-BRST transform, i.e., a relation of the same type as (10) for the chronological products

$$
T_{\text {anti }}^{I_{1}, \ldots, I_{n}}\left(x_{1}, \ldots, x_{n}\right) \equiv T\left(T_{\text {anti }}^{I_{1}}\left(x_{1}\right), \ldots, T_{\text {anti }}^{I_{n}}\left(x_{n}\right)\right) .
$$


Such relations should be of the form:

$$
d_{Q}^{\text {anti }} T\left(T_{\text {anti }}^{I_{1}}\left(x_{1}\right), \ldots, T_{\text {anti }}^{I_{n}}\left(x_{n}\right)\right)=i \sum_{l=1}^{n}(-1)^{s_{l}} \frac{\partial}{\partial x_{l}^{\mu}} T\left(T_{\text {anti }}^{I_{1}}\left(x_{1}\right), \ldots, T_{\text {anti }}^{I_{l} \mu}\left(x_{l}\right), \ldots, T_{\text {anti }}^{I_{n}}\left(x_{n}\right)\right) .
$$

The hope would be to obtain new constraints on the possible anomalies. We have succeeded to study the tree contribution in the second order of perturbation theory, i.e., the preceding relation for $n=2$ and tree contributions. We have obtained the following result.

Theorem 5. We can impose the relation

$$
\begin{array}{r}
d_{Q}^{\text {anti }} T_{(0)}\left(T_{\text {anti }}^{I}\left(x_{1}\right), T_{\text {anti }}^{J}\left(x_{2}\right)\right) \\
-i \frac{\partial}{\partial x_{1}^{\mu}} T_{(0)}\left(T_{\text {anti }}^{I \mu}\left(x_{1}\right), T_{\text {anti }}^{J}\left(x_{2}\right)\right)-i(-1)^{|I|} \frac{\partial}{\partial x_{2}^{\mu}} T_{(0)}\left(T_{\text {anti }}^{I}\left(x_{1}\right), T_{\text {anti }}^{J \mu}\left(x_{2}\right)\right)=0
\end{array}
$$

for the tree components of the chronological products if we perform the following finite renormalizations:

$$
T_{(0)}\left(T_{\text {anti }}^{I}\left(x_{1}\right), T_{\text {anti }}^{J}\left(x_{2}\right)\right) \rightarrow T_{(0)}\left(T_{\text {anti }}^{I}\left(x_{1}\right), T_{\text {anti }}^{J}\left(x_{2}\right)\right)-\delta\left(x_{1}-x_{2}\right) N^{I J}\left(x_{1}\right)
$$

with the explicit expressions:

$$
\begin{gathered}
N^{[\mu v] \varnothing}=\frac{i}{2} f_{a b e} f_{c d e} \tilde{u}_{a} \tilde{u}_{b} v_{c}^{\mu} v_{d}^{v} \\
N^{[\mu][v]}=-N^{[\mu v] \varnothing} \\
N^{[\mu] \varnothing}=g_{a b c d}^{(1)} \tilde{u}_{a} v_{b}^{\mu} v_{c}^{v} v_{d v}+g_{a b c d}^{(2)} u_{a} \tilde{u}_{b} \tilde{u}_{c} v_{d}^{\mu}+g_{a b c d}^{(3)} \tilde{u}_{a} v_{b}^{\mu} \Phi_{c} \Phi_{d}
\end{gathered}
$$

with

$$
\begin{array}{r}
g_{a b c d}^{(1)}=-\frac{i}{2}\left(f_{a c e} f_{b d e}+f_{a d e} f_{b c e}\right) \\
g_{a b c d}^{(2)}=-\frac{i}{2} f_{a d e} f_{b c e} \\
g_{a b c d}^{(3)}=-\frac{i}{2}\left(f_{c e a}^{\prime} f_{e d b}^{\prime}+f_{d e a}^{\prime} f_{e c b}^{\prime}\right)
\end{array}
$$

and

$$
N^{\varnothing \varnothing}=h_{a b c d}^{(1)} v_{a \mu} v_{b}^{\mu} v_{c}^{v} v_{d v}+h_{a b c d}^{(2)} v_{a}^{\mu} v_{b \mu} \Phi_{c} \Phi_{d}+\frac{1}{4 !} h_{a b c d}^{(3)} \Phi_{a} \Phi_{b} \Phi_{c} \Phi_{d}
$$

with

$$
\begin{array}{r}
h_{a b c d}^{(1)}=-\frac{i}{4}\left(f_{a d e} f_{b c e}+f_{a c e} f_{b d e}\right) \\
h_{a b c d}^{(2)}=-\frac{i}{2}\left(f_{d e a}^{\prime} f_{e c b}^{\prime}+f_{c e a}^{\prime} f_{e d b}^{\prime}\right) \\
h_{a b c d}^{(3)} m_{a}=-2 i\left(f_{e b a}^{\prime} f_{e c d}^{\prime \prime}+f_{e c a}^{\prime} f_{e b d}^{\prime \prime}+f_{e d a}^{\prime} f_{e b c}^{\prime \prime}\right) .
\end{array}
$$

Only the linear and bilinear constraints from the end of the Section 2.4 are needed for this result. The finite renormalization (53) is identical to the finite renormalization needed for the usual gauge invariance of the tree contributions in the second order of perturbation theory.

Proof. (i) We start with the case $I=[\mu v], J=\varnothing$ of identity (46). We start from the identity

$$
\left.d_{Q}^{\text {anti }}\left[T_{\text {anti }}^{\mu v}\left(x_{1}\right), T\left(x_{2}\right)\right)\right]-i \frac{\partial}{\partial x_{2}^{\rho}}\left[T_{\text {anti }}^{\mu v}\left(x_{1}\right), T_{\text {anti }}^{\rho}\left(x_{2}\right)\right]=0
$$

and do the substitution (34) $\rightarrow$ (35). We need to collect all the terms from the commutator $\left[T_{\text {anti }}^{\mu v}\left(x_{1}\right), T_{\text {anti }}^{\rho}\left(x_{2}\right)\right]$ containing the factor $\partial^{\rho} D\left(x_{1}-x_{2}\right)$. The identity (55) holds if we use 
the Klein-Gordon equation for the Pauli-Villars distribution $\left(\square+m^{2}\right) D_{m}=0$. However, when we make the substitution (34) $\rightarrow$ (35) we must use $\left(\square+m^{2}\right) D_{m}^{F}=\delta$ and an anomaly appears. For instance, if we consider the first term of $T_{\text {anti }}^{\rho}\left(x_{2}\right)$ - see the expression (42)we have

$$
\begin{array}{r}
{\left[T_{\text {anti }}^{\mu v}\left(x_{1}\right), T_{\text {anti }}^{\rho}\left(x_{2}\right)_{1}\right]=\frac{1}{2} f_{a b c} f_{p q r}\left[\left(\tilde{u}_{a} \tilde{u}_{b} F_{c}^{\mu v}\right)\left(x_{1}\right),\left(\tilde{u}_{p} v_{q \sigma} F_{c}^{\sigma \rho}\right)\left(x_{2}\right)\right]} \\
=\frac{i}{2} f_{a b e} f_{c d e}\left[\partial^{\mu} \partial^{\rho} D_{m_{c}}\left(x_{1}-x_{2}\right)\left(\tilde{u}_{a} \tilde{u}_{b}\right)\left(x_{1}\right),\left(\tilde{u}_{c} v_{d}^{v}\right)\left(x_{2}\right)-(\mu \leftrightarrow v)\right]+\cdots
\end{array}
$$

and after the substitution (34) $\rightarrow$ (35) we obtain the anomaly

$$
A_{1}^{\mu v}=-\frac{1}{2} f_{a b e} f_{c d e}\left[\partial^{\mu} \delta\left(x_{1}-x_{2}\right)\left(\tilde{u}_{a} \tilde{u}_{b}\right)\left(x_{1}\right)\left(\tilde{u}_{c} v_{d}^{v}\right)\left(x_{2}\right)-(\mu \leftrightarrow v)\right]
$$

We have another term from the fourth term of $T_{\text {anti }}^{\rho}\left(x_{2}\right)$ and it is convenient to exhibit the end result in the form:

$$
A^{\mu v}\left(x_{1}, x_{2}\right)=\delta\left(x_{1}-x_{2}\right) a^{\mu v}\left(x_{1}\right)+\partial_{\rho} \delta\left(x_{2}-x_{1}\right) a^{\mu v ; \rho}\left(x_{1}\right) .
$$

After some work, using Jacobi identity, we obtain that

$$
a^{\mu v ; \rho}=0 \quad a^{\mu v}=d_{Q}^{\text {anti }} N^{[\mu v] \varnothing}
$$

where $N^{[\mu v] \varnothing}$ is the expression from the statement. This anomaly can be eliminated by performing the finite renormalization (49).

(ii) Now we consider the identity

$$
\left.d_{Q}^{\text {anti }}\left[T_{\text {anti }}^{\mu}\left(x_{1}\right), T_{\text {anti }}^{v}\left(x_{2}\right)\right)\right]-i \frac{\partial}{\partial x_{1}^{\rho}}\left[T_{\text {anti }}^{\mu \rho}\left(x_{1}\right), T_{\text {anti }}^{v}\left(x_{2}\right)\right]+i \frac{\partial}{\partial x_{2}^{\rho}}\left[T_{\text {anti }}^{\mu}\left(x_{1}\right), T_{\text {anti }}^{v \rho}\left(x_{2}\right)\right]=0 .
$$

Now we need to collect all the terms from the two commutators $\left[T_{\text {anti }}^{\mu \rho}\left(x_{1}\right), T_{\text {anti }}^{\nu}\left(x_{2}\right)\right]$ and $\left[T_{\text {anti }}^{\mu}\left(x_{1}\right), T_{\text {anti }}^{v \rho}\left(x_{2}\right)\right]$ containing the factor $\partial^{\rho} D\left(x_{1}-x_{2}\right)$; afterwards we make the substitution (34) $\rightarrow$ (35) and obtain the corresponding anomaly. The computation leads, after using Jacobi identity, to the anomaly

$$
B^{\mu v}\left(x_{1}, x_{2}\right)=\delta\left(x_{1}-x_{2}\right) b^{\mu v}\left(x_{1}\right)
$$

where

$$
b^{\mu v}=d_{Q}^{\mathrm{anti}} N^{[\mu][v]}
$$

with $N^{[\mu][v]}$ the expression from the statement. This anomaly can be eliminated by performing the finite renormalization (50).

(iii) The next step is the identity

$$
\left.d_{Q}^{\text {anti }}\left[T_{\text {anti }}^{\mu}\left(x_{1}\right), T\left(x_{2}\right)\right)\right]-i \frac{\partial}{\partial x_{1}^{v}}\left[T_{\text {anti }}^{\mu v}\left(x_{1}\right), T\left(x_{2}\right)\right]+i \frac{\partial}{\partial x_{2}^{v}}\left[T_{\text {anti }}^{\mu}\left(x_{1}\right), T_{\text {anti }}^{v}\left(x_{2}\right)\right]=0 .
$$

There are a lot of terms with the factor $\partial^{v} D\left(x_{1}-x_{2}\right)$ contributing to the anomaly. There is another subtlety: in the right hand side we have to use in fact the chronological products renormalized according to (49) and (50). This brings additional contributions to the anomaly:

$$
i \frac{\partial}{\partial x_{1}^{\nu}}\left[\delta\left(x_{1}-x_{2}\right) N^{[\mu v] \varnothing}\left(x_{1}\right)\right]-i \frac{\partial}{\partial x_{2}^{\nu}}\left[\delta\left(x_{1}-x_{2}\right) N^{[\mu][v]}\left(x_{1}\right)\right] .
$$


We must use all the bilinear identities (29)-(32) from the end of Section 2 and end up with an anomaly of the type

$$
C^{\mu}\left(x_{1}, x_{2}\right)=\delta\left(x_{1}-x_{2}\right) c^{\mu}\left(x_{1}\right)
$$

where the expression for $c^{\mu}$ is rather complicated. We try to equate it with an expression of the type $d_{Q}^{\text {anti }} N^{\mu}$ where $N^{\mu}$ generic form (51). We end up with a system of four equations: from three of them the expressions of $g^{(j)}, j=1,2,3$ from the statement are derived and the fourth is an identity if we use the bilinear identities (29)-(32). So, this anomaly can, again, be eliminated by a finite renormalization (51).

(iv) This repetitive process ends with the consideration of the identity:

$$
\left.d_{Q}^{\text {anti }}\left[T\left(x_{1}\right), T\left(x_{2}\right)\right)\right]-i \frac{\partial}{\partial x_{1}^{\mu}}\left[T_{\text {anti }}^{\mu}\left(x_{1}\right), T\left(x_{2}\right)\right]-i \frac{\partial}{\partial x_{2}^{\mu}}\left[T\left(x_{1}\right), T_{\text {anti }}^{\mu}\left(x_{2}\right)\right]=0 .
$$

As before, we select the terms from the commutators with the factor $\partial^{\mu} D\left(x_{1}-x_{2}\right)$. They will produce a piece of the anomaly after the substitution (34) $\rightarrow$ (35). There is another piece coming from the finite renormalization (51):

$$
i \frac{\partial}{\partial x_{1}^{\mu}}\left[\delta\left(x_{1}-x_{2}\right) N^{[\mu] \varnothing}\left(x_{1}\right)\right]+\left(x_{1} \longleftrightarrow x_{2}\right) .
$$

If we use the bilinear identities (29)-(32) from the end of Section 2 and end up with an anomaly of the type

$$
D\left(x_{1}, x_{2}\right)=\delta\left(x_{1}-x_{2}\right) d\left(x_{1}\right)
$$

where the expression for $d$ is complicated. We try to equate it with an expression of the type $d_{Q}^{\text {anti }} N$ where $N$ generic form (53). We end up with a system of four equations: from three of them the expressions of $h^{(j)}, j=1,2,3$ from the statement are derived and the fourth is an identity if we use the bilinear identities (29)-(32). So, this anomaly can, again, be eliminated by a finite renormalization (53).

We end with an explanation of the fact that we obtain nothing new from the anti-BRST transform. In fact, anti-BRST is equivalent to BRST transform. One can see this if replaces the interaction Lagrangian $T$ from (20) by an equivalent Lagrangian, i.e., an expression $T^{\prime}$ differing from $T$ by a co-boundary. In fact, we can rewrite the second term from (20) in the following way:

$$
T_{2}=\frac{1}{2} f_{a b c}\left(u_{a} v_{b}^{\mu} \partial_{\mu} \tilde{u}_{c}-\partial_{\mu} u_{a} v_{b}^{\mu} \tilde{u}_{c}-m_{a} \Phi_{a} \tilde{u}_{b} u_{c}\right)+d_{Q} B+\partial_{\mu} B^{\mu}
$$

where we omit the explicit expressions $B$ and $B^{\mu}$. It is known that we can discard the co-boundary $d_{Q} B+\partial_{\mu} B^{\mu}$ without modifying the values of scattering matrix taken in the subspace of the physical states [29]. We can prove that the new interaction Lagrangian obtained in this way is anti-symmetric with respect to the transform (44). This antisymmetry property can be extended to higher order chronological products, so if we have gauge invariance with respect to the BRST transform, then we must have gauge invariance with respect to the anti-BRST transform also.

\section{Conclusions}

The main point of this note is that the elimination of the anomalies in higher orders of perturbation theory is an extremely difficult problem. All simple ideas, like for instance, the use of new symmetries, as the anti-BRST symmetry, do not produce new constraints on the anomalies.

Funding: The author acknowledges the financial support received from the Romanian Ministry of Research, Digitalisation and Innovation through the Project PN 1906 01 01/2019. 
Institutional Review Board Statement: Not applicable.

Informed Consent Statement: Not applicable.

Data Availability Statement: Not applicable.

Acknowledgments: The author acknowledges the financial support received from the Romanian Ministry of Research, Digitalisation and Innovation through the Project PN 190601 01/2019.

Conflicts of Interest: The author declares no conflict of interest.

\section{References}

1. Bogoliubov, N.N.; Shirkov, D. Introduction to the Theory of Quantized Fields, 3rd ed.; John Wiley and Sons: Hoboken, NJ, USA, 1976.

2. Duetsch, M.; Fredenhagen, K. A Local (Perturbative) Construction of Observables in Gauge Theories: The Example of QED. Commun. Math. Phys. 1999, 203, 71-105. [CrossRef]

3. Epstein, H.; Glaser, V. The Rôle of Locality in Perturbation Theory. Ann. Inst. H. Poincaré 1973, 19, $211-295$.

4. Glaser, V. Electrodynamique Quantique. L'enseignement du 3e Cycle de la Physique en Suisse Romande (CICP), Semestre d'hiver $1972 / 73$.

5. Scharf, G. Finite Quantum Electrodynamics: The Causal Approach, 2nd ed.; Springer: Berlin/Heidelberg, Germany, 1995.

6. Scharf, G. Gauge Field Theories: Spin One and Spin Two: 100 Years after General Relativity; Courier Dover Publications: Mineola, NY, USA, 2010.

7. Stora, R. Lagrangian Field Theory; Les Houches Lectures; Witt, C.D., Itzykson, C., Eds.; Gordon and Breach: Philadelphia, PA, USA, 1971.

8. Popineau, G.; Stora, R. A Pedagogical Remark on the Main Theorem of Perturbative Renormalization Theory. Nucl. Phys. B 2016, 912, 70-78. [CrossRef]

9. Steinmann, O. Perturbation Expansions in Axiomatic Field Theory; Lecture Notes in Physics; Springer: Berlin/Heidelberg, Germany, 1971; Volume 11.

10. Duetsch, M. From Classical Field Theory to Perturbative Quantum Field Theory; Progress in Mathematical Physics; Springer: Berlin/Heidelberg, Germany, 2019; Volume 74.

11. Rejzner, K. Perturbative Algebraic Quantum Field Theory: An Introduction for Mathematicians; Springer: Berlin/Heidelberg, Germany, 2016.

12. Gracia-Bondia, J.M. The Epstein-Glaser Approach to QFT. AIP Conf. Proc. 2006, 809, $24-43$.

13. Gracia-Bondia, J.M. Lectures on BRS Invariance for Massive Boson Fields. arXiv 2008, arXiv:0808.2853v2.

14. Hollands, S. Renormalized Quantum Yang-Mills Fields in Curved Spacetime. Rev. Math. Phys. 2008, 20, 1033-1172. [CrossRef]

15. Schroer, B. Peculiarities of Massive Vector Mesons and Their Zero Mass Limits: To the Memory of Raymond Stora. Eur. Phys. J. C 2015, 75, 365. [CrossRef]

16. Ojima, I. Another BRS Transformation. Prog. Theor. Phys. 1980, 64, 625-638. [CrossRef]

17. Chryssomalakos, C.; de Azcárraga, J.A.; Macfarlane, A.J.; Bueno, J.C.P. Higher-order BRST and anti-BRST operators and cohomology for compact Lie algebras. J. Math. Phys. 1999, 40, 6009-6032. [CrossRef]

18. Barnich, G.; Henneaux, M. Renormalization of Gauge Invariant Operators and Anomalies in Yang-Mills Theory. Phys. Rev. Lett. 1994, 72, 1588-1591 [CrossRef]

19. Bilal, A. Lectures on Anomalies. arXiv 2008, arXiv:0802.0634.

20. Grigore, D.R. Cohomological Aspects of Gauge Invariance in the Causal Approach. Rom. J. Phys. 2010, 55, 386-438.

21. Grigore, D.R. On the Uniqueness of the Non-Abelian Gauge Theories in Epstein-Glaser Approach to Renormalisation Theory. Rom. J. Phys. 1999, 44, 853-913.

22. Grigore, D.R. The Standard Model and its Generalisations in Epstein-Glaser Approach to Renormalisation Theory. J. Phys. A 2000, 33, 8443-8476. [CrossRef]

23. Grigore, D.R. The Standard Model and its Generalisations in Epstein-Glaser Approach to Renormalisation Theory II: The Fermion Sector and the Axial Anomaly. J. Phys. A 2001, 34, 5429-5462. [CrossRef]

24. Duetsch, M.; Scharf, G. Perturbative gauge invariance: The electroweak theory. Ann. Phys. (Leipzig) 1999, 8, 359-387. [CrossRef]

25. Scharf, G. General massive gauge theory. Il Nuovo Cimento A 1999, 112, 619-638. [CrossRef]

26. Duch, P.; Duetsch, M.; Gracia-Bondia, J.M. Diphoton decay of the Higgs from the Epstein-Glaser viewpoint. arXiv 2020, arXiv:2011.12675.

27. Duetsch, M. Massive vector bosons: Is the geometrical interpretation as a spontaneously broken gauge theory possible at all scales? arXiv 2015, arXiv:1501.02599v3.

28. Duetsch, M.; Gracia-Bondia, J.M.; Scheck, F.; Varilly, J.C. Quantum gauge models without classical Higgs mechanism. Eur. Phys. J. C 2010, 69, 599-621. [CrossRef]

29. Grigore, D.R. Trivial Lagrangians in the Causal Approach. Rom. J. Phys. 2016, 61, 320-332. 